of IgAV patients from two Croatian University Centers for pediatric rheumatology.

81 pediatric IgAV patients were included, of whom 45 were boys and 36 girls, as well as 150 age- and sex-matched healthy controls without any history of autoimmune disease. The median (range) age of IgAV patients was 6.25 (4.60-8.20) years, and among them $71.6 \%$ had joint involvement, $29.62 \%$ had gastrointestinal manifestations, while $27.16 \%$ developed nephritis. The purpuric rash which extended from lower extremities to the trunk, upper extremities and face (generalized rash) was present in $43.20 \%$ of patients and $27.16 \%$ had at least one relapse. Among the analyzed polymorphisms, only in the rs1412125 there was a deviation from the Hardy Weinberger equilibrium. There was no statistically significant association of the analyzed polymorphisms with the IgAV susceptibility, compared to healthy controls. Polymorphism rs2070600 was significantly related with the development of nephritis in IgAV, while rs1412125 was associated with gastrointestinal involvement. The IgAV patients carrying the $\mathrm{T}$ allele (rs2070600) of the AGER had significantly increased risk of nephritis development compared with the IgAV patients with homozygous CC genotype in dominant (OR 4.05, CI 1.0915.03, $\mathrm{p}=0.037$ ) and additive genetic models (OR 3.95, CI 1.16-13.47, $\mathrm{p}=0.049$ ). The minor $\mathrm{C}$ allele (rs1412125) of the HMGB1 was found to significantly increase the risk of gastrointestinal involvement in overdominant model with an allelic odd ratio of 2.78 (CI 1.04-7.43, p $=0.04$ ).

Although neither of analyzed HMGB1 and RAGE polymorphisms was not associated with IgAV susceptibility, our results indicated that these polymorphisms may be involved in the pathogenesis of IgAV with possible effect on different disease phenotypes.

SUPPORT: Croatian Science Foundation project IP-2019-048822.

\section{SYSTEMIC JUVENILE IDIOPATHIC ARTHRITIS WITHOUT ARTHRITIS AS THE CAUSE OF INTERMITTENT PERSISTENT FEVER IN A 7-YEAR OLD GIRL}

${ }^{1}$ Ana Smajo*, 'Vana Vukić, ${ }^{1,2}$ Lovro Lamot. 'University of Zagreb, School of Medicine; ${ }^{2}$ Division of Clinical Immunology and Rheumatology, Department of Pediatrics, Clinical Hospital Center Sestre Milosrdnice, Zagreb, Hrvatska

10.1136/archdischild-2021-europaediatrics.447

Background Intermittent fever along with increased inflammatory markers (CRP and ESR) most commonly is the sign of an infectious disease. Nevertheless, without an adequate response to antibiotic treatment, it should raise concern of other conditions, including several rheumatic, like Kawasaki disease, periodic fever syndromes and systemic form of juvenile idiopathic arthritis (JIA), even when no clear arthritis is present.

Case Presentation We present a case of a 7-year-old girl with a 14-month history of intermittent fever with increased inflammatory markers, along with unspecific symptoms such as hepatomegaly, polymorphous rash and migratory arthralgia. An extensive diagnostic workup excluded infectious aetiology, genetic testing did not detect pathogenic mutations. Despite the treatment with intravenous imunoglobulines and low dose glucocorticoids (GCs), the fever did not subside. Finally, extensive laboratory workup revealed increased proinflammatory cytokines IL-6 and TNF-alpha along with chronic anaemia and thrombocytosis. The systemic subtype of JIA was considered, and treatment with pulse $(30 \mathrm{mg} / \mathrm{kg})$, continued with high $(2 \mathrm{mg} / \mathrm{kg})$ doses of GCs was initiated with an instant resolution of symptoms. Nevertheless, after the weaning of GCs, the new exacerbation was observed and therefore tocilizumab, humanized monoclonal antibody against IL-6 receptor, was initiated.

Conclusion Systemic form of juvenile idiopathic arthritis is a heterogeneous disease dominated musculoskeletal and systemic symptoms. While the former ones are a result of similar pathophysiologiocal mechanisms as other forms of JIA, activation of the nonspecific immune response is responsible for the sytemic ones, similar to other autoinflammatory diseases. Therefore, it is possible that in some cases of systemic form of JIA, systemic inflammation is present without the musculoskeletal symptoms. With the existing classification criteria of the International League against rheumatism (ILAR) it can be difficult to diagnose and initiate appropriate therapy. Consequently it can mislead to recognize a serious complication macrophage activation the syndrome. The recognition of the unique nature of systemic JIA in comparison to other types of JIA as well as an increased understanding of its pathogenesis, provides a better outcome and prognosis for children who often go undiagnosed with a debilitating chronic condition.

\section{\begin{tabular}{|l|l}
\hline 448 & TREATMENT OF JUVENILE SPONDYLOARTHRITIS
\end{tabular}}

${ }^{1}$ Vana Vukić ${ }^{*},{ }^{2}$ Mandica Vidović, ${ }^{1,2}$ Miroslav Harjaček, ${ }^{1,2}$ Lovro Lamot. ${ }^{1}$ School of Medicine University of Zagreb; ${ }^{2}$ Department of Clinical Immunology and Rheumatology, Department of Pediatrics, University Hospital Centre Sestre milosrdnice, Zagreb, Croatia

10.1136/archdischild-2021-europaediatrics.448

Juvenile spondyloarthritis (jSpA) represents a spectrum of inflammatory arthritis with strong HLA-B27 association and involvement of enthesis and/or axial skeleton that appears in children and young adults. ILAR classification criteria for enthesitis related arthritis subtype of juvenile idiopathic arthritis, which is undifferentiated form of jSpA, includes arthritis, enthesitis, presence of sacroiliac joint tenderness, inflammatory lumbosacral pain, HLA-B27 positivity, positive family history and acute anterior uveitis.

We present a case of 16 years old girl diagnosed with juvenile spondyloarthritis that first presented with recurrent monthly swelling of index finger at the age of 14 . Symptoms progressed to low back pain in the morning which partly declined with activity. Additionally, her right knee was swollen and painful. She was examined on multiple occasions by pediatric orthopedic surgeon and in pediatric emergency department before seeing a pediatric rheumatologist. The first examination revealed sacroiliac joint tenderness with positive FABER test and abnormal modified Schober test. Family history was negative for rheumatic diseases and there were no signs of uveitis nor enthesitis, with ANA, RF and extensive laboratory workup being either negative or within reference range. However, HLA-B27 turned positive and MRI showed right sacroiliitis. NSAID was prescribed but symptoms nevertheless persisted. Thus, after the exclusion of TBC with quantiferon test, intraarticular corticosteroid injection was applied to the right knee and oral corticosteroid was introduced as 
bridging therapy. Subsequently, according to ACR guidelines, a therapy with TNF-alpha inhibitor, adalimumab, was initiated. Within a month, a substantial reduction of the low back pain was noted, with a decrease in juvenile spondyloarthritis disease activity score (jSpADA).

Low back pain often begins in childhood, with the prevalence in adolescence being similar to that in adulthood. Among many possible causes, inflammatory etiology should be thoroughly considered due to irreversible damage if not treated adequately. Therefore, children and adolescents complaining of a back pain, especially in the presence of other signs of arthritis, should be referred to pediatric rheumatologist for a further work-up and treatment.

\section{THE ART OF RECOGNIZING PAIN AMPLIFICATION SYNDROME IN CHILDREN}

${ }^{1}$ Vana Vukić*, ${ }^{2}$ Mandica Vidović, ${ }^{1,2}$ Miroslav Harjaček, ${ }^{1,2}$ Lovro Lamot. ${ }^{1}$ Institution of the presenting author; ${ }^{2}$ School of Medicine University of Zagreb;, Department of clinical immunology and rheumatology, Department of Pediatrics, University Hospital Centre Sestre milosrdnice, Zagreb, Croatia

\subsection{6/archdischild-2021-europaediatrics.449}

Juvenile fibromyalgia is a pain amplification syndrome characterized by diffuse idiopathic musculoskeletal pain, most frequently affecting children older than 10 years. Girls are more commonly affected than boys, with the ratio of $1: 4-8$. American College of Rheumatology criteria for diagnosis include absence of other disorder that could explain the pain, symptoms lasting for $\geq 3$ months, widespread pain index (WPI) $\geq 7$ and symptom severity score (SSS) $\geq 5$ or WPI $3-6$ and SSS $\geq 9$.

We present a case of 15 years old girl with diffuse and persistent pain along with episodes of impaired consciousness. Those episodes occurred once a month for the last 3 years and were characterized by collapse and/or inability to move or speak for several minutes. They were preceded by paraesthesia of face, arms and feet, but have never resulted in injury. In addition, patient complained of pain in radiocarpal joints, hips, low back, groins and neck, along with extreme tiredness, poor sleeping and inability to concentrate.

Extensive examination was undertaken. The neurological assessment included brain MRI and EEG, both of which were normal. The rheumatologic evaluation revealed multiple painful points with WPI 9 and SSS 8. The patient didn't experience morning stiffness, joint swelling or fever. Extensive laboratory workup and MRI of sacroiliac joints along with a lumbar and thoracic portion of the spine excluded the inflammatory aetiology. The psychological assessment revealed poor stress coping strategies. The patient was diagnosed with fibromyalgia and conversion disorder manifested in the form of pseudoseizures. Physical therapy and psychotherapy were advised, along with melatonin for better sleep regulation, which led to a mild improvement of symptoms within a month.

Fibromyalgia and diverse psychological distress are causally related. Accordingly, conversion symptoms are not uncommon. Therefore, a multidisciplinary team approach is necessary when caring for these patients, in addition to providing treatment not only for the affected individual but for the entire family as well.

\section{KAWASAKI DISEASE WITH PULMONARY MANIFESTATIONS - A CASE REPORT OF THREE PATIENTS}

${ }^{1}$ Ivana Sosa Filjak*, ${ }^{2}$ Maja Batinica, ${ }^{2,3}$ Arnes Rešić, ${ }^{2,4}$ Alenka Gagro. ${ }^{1}$ Institution of the presenting author; 'General Hospital Zabok; ' ${ }^{2}$ Clinic for Pediatrics, Clinic for Children's Diseases Zagreb, Faculty of Medicine, University of Zagreb; ${ }^{3}$ Faculty of Medicine, University of Split; ${ }^{4}$ Faculty of Medicine, Josip Juraj Strossmayer University of Osijek

\subsection{6/archdischild-2021-europaediatrics.450}

Kawasaki disease (KD) represents systematic vasculitis of unknown etiology that typically occurs in childhood. Its most important complications are coronary artery changes described in 15 to $25 \%$ of untreated children. The diagnosis is based on fever criteria longer than five days and at least four additional criteria: polymorphic exanthema, changes in the extremities, mucosal changes in the oral cavity or on the lips, bilateral conjunctival injection and unilateral cervical lymphadenopathy. Affection of lungs is rare manifestation in $\mathrm{KD}$ and can present as pneumonia, pulmonary nodules, bronchopneumonia, hydropneumothorax and pleural effusion. During a ten year period, we treated 11 children diagnosed with KD in our clinic, of which $\mathrm{KB}$ presented with clinical and image-proven lung involvement during the acute phase.

CASE REPORT: Patient 1.: A 16 year old adolescent presented with persistent fever, cough, macular rash of the trunk and extremities, conjunctival injection and hepatosplenomegaly. Bilateral interstitial pneumonia with bilateral pleural effusion was radiologically confirmed. An MSCT of thorax was performed, in which nodular confluent consolidations and ground glass opacities, along with pleural effusion, were described. No cardiological manifestations occurred during acute phase or during follow up of the patient.

Patient 2.: A 6 year old boy presented with fever, abdominal pain, haemorrhagic diarrhoea, oedema of the eyelids, feet and hands, conjunctival injection and maculo-papular rash in the lower abdomen. Radiological and ultrasound findings showed alveolo-interstitial pneumonia with bilateral pleural effusion. Cardiological processing proved myocarditis, fusiform aneurysm of the right coronary artery and dilatation of the left coronary artery.

Patient 3.: A 5 year old girl presented with prolongated fever, inguinal lymphadenitis, oedema of the eyelids and lips, conjunctival injection, macular rash on the trunk and extremities and erythema multiforme on the abdomen. Chest x-ray and ultrasound verified right-sided supradiaphragmatic infiltration with minor bilateral pleural effusion. The course od the disease was complicated by multiorgan failure syndrome and the development of $\mathrm{KD}$ associated shock syndrome. Echocardiography verified changes on both coronary arteries.

Microbiological analysis was negative in all patients with $\mathrm{KB}$ and pulmonary manifestations. Pulmonary manifestations did not improve upon administration of antibiotic treatment. All patients answered positive to treatment with intravenous immunoglobulins and acetylsalicylic acid. Corticosteroids and additional intravenous immunoglobulins were administered in patient with KD associated shock.

Conclusion According to the literature, pulmonary manifestations have been described in 20 patients (1). Of these, seven had coronary artery changes, 10 patients had incomplete disease and seven patients requested an additional, second dose of intravenous immunoglobulins with or without corticosteroids. Although the etiology of pulmonary manifestations in 